University of Nebraska - Lincoln DigitalCommons@University of Nebraska - Lincoln

CSE Conference and Workshop Papers

Computer Science and Engineering, Department of

2014

\title{
Network Path Capacity Comparison without Accurate Packet Time Information
}

Ertong Zhang

University of Nebraska-Lincoln, ezhang@cse.unl.edu

Lisong $\mathrm{Xu}$

University of Nebraska-Lincoln, xu@cse.unl.edu

Follow this and additional works at: http:// digitalcommons.unl.edu/cseconfwork

Zhang, Ertong and Xu, Lisong, "Network Path Capacity Comparison without Accurate Packet Time Information" (2014). CSE

Conference and Workshop Papers. 254.

http://digitalcommons.unl.edu/cseconfwork/254

This Article is brought to you for free and open access by the Computer Science and Engineering, Department of at DigitalCommons@University of Nebraska - Lincoln. It has been accepted for inclusion in CSE Conference and Workshop Papers by an authorized administrator of

DigitalCommons@University of Nebraska - Lincoln. 


\title{
Network Path Capacity Comparison without Accurate Packet Time Information
}

\author{
Ertong Zhang and Lisong Xu \\ Department of Computer Science and Engineering \\ University of Nebraska-Lincoln \\ Lincoln, NE 68588-0115 \\ Email: \{ezhang, xu\}@cse.unl.edu
}

\begin{abstract}
A fundamental problem of current bandwidth estimation methods is that they require accurate packet time information. However, it is hard to accurately measure packet time information in an increasing number of network environments, such as widely deployed highspeed networks, and emerging cloud computing networks. Motivated by the observation that many applications only need the relative bandwidth information of different paths instead of the actual bandwidth information of a single path, we propose sequence-based bandwidth comparison. Specifically, this paper proposes a capacity comparison method, called PathComp, which can relatively compare the capacities of the paths from two senders to the same receiver. PathComp mainly uses the arrival sequence information of packets, and does not require any accurate packet time information. Our testbed, campus network, and EC2 experiments show that PathComp can not only determine which path is faster but also accurately determine how much faster in a variety of network environments.
\end{abstract}

Index Terms-Internet measurement; Bandwidth estimation; Capacity estimation.

\section{INTRODUCTION}

A rich body of bandwidth estimation methods [20] have been proposed and studied in the past two decades, due to the wide range of applications of bandwidth estimation. However, there is a fundamental problem with the current bandwidth estimation methods. Most (if not all) of them need to accurately measure certain time information of network packets, such as the arrival time difference (ATD) between two consecutive packets [6], the one-way or round-trip delay of each packet [10], and the queueing delay of each packet [8]. However, it is hard and sometimes impossible to accurately measure these time information in an increasing number of network environments, such as widely deployed highspeed networks, and emerging cloud computing networks.

There are two major reasons why it is sometimes hard to accurately measure the packet time information. First, it takes very short times to send or receive packets at very high speeds. However, it is hard to measure such short times due to the limited system capability [9], [17]. Second, various software and hardware factors at the receiver of packets, such as interrupt moderation [19], [9] (commonly used in highspeed network cards) and virtual machine (VM) scheduling [21], [4] (commonly used in cloud computing), greatly change the original packet time information. As a result, the packet time information measured by the packet receiver is not correct.

Our work is motivated by the observation that many applications only need to relatively compare the bandwidth information of different paths. For example, in a peer-to-peer (P2P) network, a new peer needs to select several fastest peers as its neighbors from a set of existing peers. More motivating examples will be discussed in Section II-A. In these cases, we do not need to measure the actual bandwidth information of each path, instead, we only need to relatively compare the bandwidth information of different paths, and then rank them according to their bandwidth information.

In this paper, we study how to relatively compare the bandwidth information of multiple paths without requiring accurate packet time information. There are several important bandwidth metrics [20]. As the first step, this paper considers only the capacity of a path that is the capacity of the narrow link in the path, and the narrow link of a path is the link with the smallest capacity among all links in a path. The path capacity is a basic bandwidth metric and will provide useful information for studying other bandwidth metrics, such as available bandwidth and bulk TCP throughput, which will be considered in our future work.

Specifically, this paper proposes a capacity comparison method, called PathComp, which can relatively compare the path capacities from two senders to the same receiver. Basically, PathComp actively sends probing packets from both senders to the receiver, measures the arrival sequence of these packets at the receiver, and then relatively compares the capacities of the two paths.

PathComp is based on the fact that the inter-arrival gap between two consecutive packets from the same sender is related to the capacity of their path. This fact is also the basis of the current capacity estimation methods [6], [10]. The uniqueness of PathComp is that it measures the packet inter-arrival gap using the packet arrival sequence information, whereas the current capacity estimation methods measure the packet inter-arrival gap using the packet arrival time information. Therefore, PathComp does not require any accurate packet time information, and is fundamentally different from the current capacity estimation methods.

The contributions of this paper are as follows. First, we expand the design space of traditional time-based bandwidth 
estimation methods by introducing a new class of sequencebased bandwidth comparison methods. Note that bandwidth comparison methods are inherently more scalable than traditional bandwidth estimation methods in terms of the measurement time for a large number of paths. This is because bandwidth comparison methods are designed to simultaneously measure multiple paths, whereas traditional bandwidth estimation methods are designed to measure a single path and are sensitive to the interference among multiple concurrent measurements [5].

Second, we propose a capacity comparison method, called PathComp, which can determine not only which path is faster but also how much faster in terms of the path capacity. In the paper, we thoroughly study the impact of various types of cross traffic on capacity comparison, and we also discuss some implementation challenge, such as Receiver Side Scaling [14]. Our testbed, campus network, and Amazon EC2 [1] experiments show that PathComp can accurately compare the capacities of two paths in a variety of network environments.

The remainder of this paper is organized as follows: Section II gives more detailed description of our motivations. Section III summarizes the related work and discusses the design space of bandwidth estimation methods. Section IV describes the basic idea of sequence-based capacity comparison. Section V studies the impact of cross traffic. Section VI presents our proposed PathComp. Section VII shows the evaluation results. Finally, Section VIII concludes the paper.

\section{Motivation}

\section{A. Bandwidth Comparison Scenarios}

Our work is motivated by the observation that many applications only need to relatively compare the bandwidth information of different paths.

$P 2 P$ neighbor selection: When a new peer joins a $\mathrm{P} 2 \mathrm{P}$ network [13], it usually needs to select its neighbors from a set of existing peers. Typically, the new peer selects the existing peers with fast network bandwidth as its neighbors so that it can quickly download data from its neighbors. Bandwidth comparison methods can be used to quickly select several fastest peers from a set of existing peers.

Network-aware task placement [11]: Consider a bandwidthintensive cloud application with three tasks: $T_{1}, T_{2}$, and $T_{3}$, and a cloud consisting of three interconnected VMs: $V_{1}, V_{2}$, and $V_{3}$. Assume that tasks $T_{1}$ and $T_{2}$ communicate often with task $T_{3}$, but not much with each other. If we find that the path between $V_{1}$ and $V_{2}$ is the slowest one using a bandwidth comparison method, and network measurements show that the latency between any two of these three VMs is the same, then the application performance can be improved with the optimal task placement that places task $T_{3}$ on $V_{3}$, and places the other two tasks on the other two VMs.

\section{B. Difficulties in Obtaining Accurate Packet Time Information}

Another motivation of our work is that the current timebased capacity measurement algorithms do not work well in some network environments, such as highspeed networks and cloud computing networks, where it is hard to accurately measure the packet time information. There are two major reasons.

First, it takes very short times to send or receive packets at very high speeds. For example, it takes only $12 \mu \mathrm{s}$ to send or receive a 1500-byte packet at $1 \mathrm{Gbps}$, and only $1.2 \mu \mathrm{s}$ at 10 Gbps. However, it is hard to accurately measure such short times due to the limited system capability [9], [17], such as clock time resolutions, clock frequency differences between the sender and the receiver, and the system call overhead.

Second, there are various software and hardward factors at the receiver of packets, such as interrupt coalescence, context switching, and virtual machine scheduling, which change the original packet time information, and thus the packet time information measured by the packet receiver is not correct.

Interrupt coalescence (IC, also called interrupt moderation) [9], [19] is commonly used in high-speed network interface cards (NIC), and it reduces the CPU load by generating an interrupt for a group of packets instead of each packet. As a result, the packet time information, such as the ATD of two consecutive packets, is changed (could be enlarged or be reduced). Figure 1 shows the measured arrival times of 200 packets from a 10Gbps NIC with IC enabled, and we can see that these packets are handled by 6 interrupts.

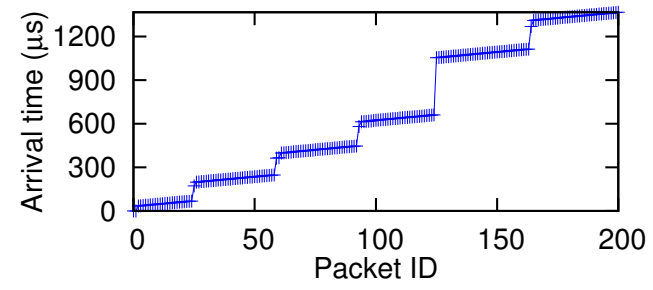

Fig. 1. Impact of interrupt coalescence on packet arrival times and ATDs.

VM scheduling [21], [4] is commonly used in cloud computing, and it enables multiple VMs to share the same pool of CPUs on a physical machine. However, it interferes with packet timestamping of VMs. For example, when a VM is not running, all packets arriving at the VM must wait until the $\mathrm{VM}$ is scheduled to run again. As a result, the packet delays and ATDs measured by the VM may be drastically different from the actual values.

\section{Design Space And Related Work}

We discuss the design space of capacity estimation methods in Figure 2, which helps us to understand the relation between the current capacity estimation methods and our proposed capacity comparison method. Some methods measure the capacity information of the path from a computer to another computer, and we refer to the first computer as the sender and the second computer as the receiver. Some other methods measure the capacity information of the round-trip path from a computer to another computer and then back to the first one. For these methods, we refer to the first computer as both the sender and receiver. 


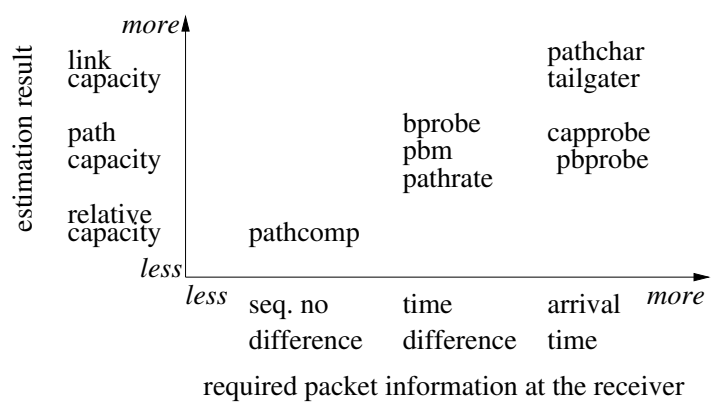

Fig. 2. The design space of capacity estimation methods.

The design space shown in Figure 2 is based on the required information of probing packets at the receiver. PathChar [7] and TailGater [12] estimate the capacity of each individual link in the path using the packet arrival times at the receiver. CapProbe [10] and PBProbe [3] estimate the path capacity using the packet arrival times. BProbe [2], PBM [16], and PathRate [6] estimate the path capacity using the packet arrival time differences at the receiver (defined in Section IV). Our proposed PathComp relatively compares the path capacities from two senders to the same receiver using the packet arrival sequence number differences at the receiver (defined in Section IV).

Note that if we know the capacity of each individual link of a path, we can infer the capacity of the path. If we know the capacities of two paths, we can infer their relative capacity ratio. Also note that the arrival times can be used to calculate the arrival time differences, and the arrival time differences can be used to infer the arrival sequence number differences. Therefore, we can see that the less the estimated capacity information, the less the required packet information.

Further more, the arrival time differences are relatively easier to accurately measure than the arrival times. For example, they are not sensitive to clock time differences between the sender and the receiver. The arrival sequence number differences can be more accurately measured than the arrival time differences. For example, they are not sensitive to the interrupt moderation at the receiver. Overall, we can see that the less the estimated capacity information, the less the required packet information, and the more robust the method.

\section{CAPACITY COMPARISON}

In this section, we explain the difference between the traditional capacity estimation problem and our proposed capacity comparison problem, and explain the difference between the traditional time-based capacity estimation methods and our proposed sequence-based capacity comparison method.

\section{A. Capacity Estimation and Comparison Problems}

We use an example illustrated in Figure 3 to describe the difference between the traditional capacity estimation problem and our proposed capacity comparison problem. There are two paths in Figure 3: path $a$ is from sender $S N D a$ to receiver $R C V$, and path $b$ is from sender $S N D b$ to the same receiver $R C V$.
Both paths merge with each other at router $R 5$. Network 5 in the figure represents everything between $R 5$ (including $R 5$ ) and $R C V$. Network 1 represents everything between $S N D a$ and the narrow link of path $a$ (called narrow link $a$ ), and network 2 for everything between narrow link $a$ and $R 5$. Similarly networks 3 and 4 for path $b$. Let $C_{a}$ denote the capacity of path $a$ that is the capacity of narrow link $a$, and let $C_{b}$ denote the capacity of path $b$ that is the capacity of narrow link $b$.

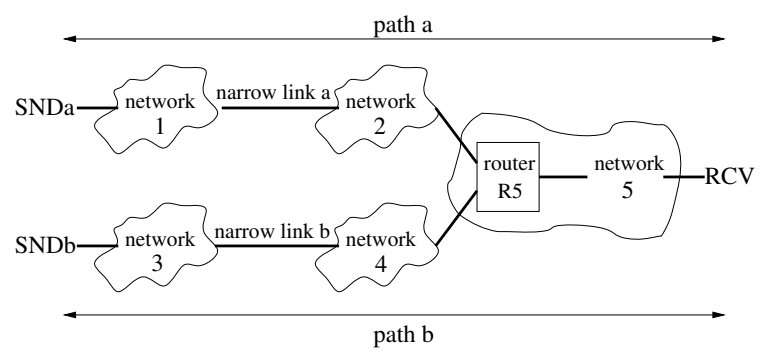

Fig. 3. Two paths: path $a$ is from sender $S N D a$ to receiver $R C V$, and path $b$ is from sender $S N D b$ to the same receiver $R C V$.

The traditional capacity estimation problem considers the capacity of the narrow link of a single path. For example, for the two paths in Figure 3, the traditional capacity estimation problem separately estimates $C_{a}$ and $C_{b}$.

Our proposed capacity comparison problem considers the capacity ratio of the narrow links of two paths. For example, for paths $a$ and $b$ in Figure 3, the capacity comparison problem estimates the capacity ratio of $C_{a}$ and $C_{b}$. That is, it relatively compares the link capacities of these two narrow links. The capacity ratio $\gamma$ of two paths $a$ and $b$ is defined as follows. Note that $\gamma$ is a real number at least 1 .

$$
\gamma= \begin{cases}C_{a} / C_{b}, & \text { if } C_{a} \geq C_{b} \\ C_{b} / C_{a}, & \text { otherwise. }\end{cases}
$$

We also define the rounded capacity ratio as follows, which is an integer at least 1 .

$$
\Gamma=\operatorname{round}(\gamma)
$$

Note that Figure 3 assumes that paths $a$ and $b$ do not have a shared narrow link (i.e., if the narrow link is located in network 5). This is a reasonable assumption for a variety of scenarios. For example, consider the P2P neighbor selection problem described in Section II-A. The narrow link of the path from a neighbor to a peer is usually the upload link of the neighbor, and thus different neighbors usually do not have a shared narrow link. As another example, consider the networkaware task placement problem in Section II-A. The narrow link of the path from a sender VM to another receiver VM is usually located near the sender VM due to the rate limiting of the sender VM, and thus the paths from different sender VMs usually do not have a shared narrow link.

In cases where two paths have a shared narrow link, there are two options. First, capacity comparison reports the capacity ratio of the narrow links of the distinct segments of the two paths. Second, capacity comparison does not report anything, if a shared narrow link is detected. However, the method to 
detect a shared narrow link is out of the scope of this paper. We choose the first option in this paper.

\section{B. Traditional Time-based Capacity Estimation}

The traditional capacity estimation methods, such as PathRate [6], and CapProbe [10], are usually based on packet arrival time differences (also called inter-arrival times, and dispersion times). The packet arrival time difference (ATD, denoted by $\tau$ ) of two packets is the time difference between their arrival times. For example, Figure 4 shows two SNDa packets, $a_{1}$ and $a_{2}$, on the link from network 5 to receiver $R C V$, and the time difference $\tau$ is their ATD at $R C V$.

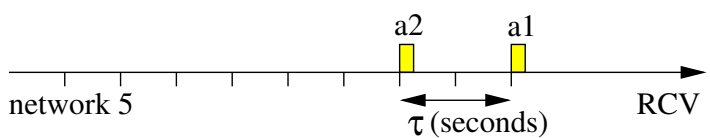

Fig. 4. The ATD $\tau$ at $R C V$ between two SNDa packets $\left(a_{1}\right.$ and $\left.a_{2}\right)$ is their arrival time difference at $R C V$.

Assuming that there is no cross traffic on path $a$, and assuming that $R C V$ can accurately measure the ATD, the capacity $C_{a}$ can be obtained as follows [6], [10], where $S$ is the packet size and $\tau$ is the ATD.

$$
C_{a}=S / \tau
$$

The traditional capacity estimation methods mainly differ in how to accurately estimate $C_{a}$ in the presence of cross traffic. However, if $R C V$ cannot accurately measure the ATD, none of these methods works.

\section{Proposed Sequence-based Capacity Comparison}

1) ASND Definition: We propose to tackle the capacity comparison problem using packet arrival sequence number differences instead of packet arrival time differences, so that our method does not require accurate packet time information. Below we use an example to explain the concept of the packet arrival sequence number differences.

Each of the two senders, $S N D a$ and $S N D b$, sends a train of $L=5$ packets of the same packet size $S$ to the receiver $R C V$ at approximately the same time. These packets are sent back-to-back by their senders (i.e., at their maximum rates). In this example, we assume that there is no cross traffic in all 5 networks in Figure 3.

The top line of Figure 5 shows the 5 SNDa packets on the link from network 2 to router $R 5$. Since there is no cross traffic, the ATD between every two consecutive SNDa packets at router $R 5$ is inversely proportional to the capacity $C_{a}$ of narrow link $a$. The bottom line of Figure 5 shows the $5 S N D b$ packets on the link from network 4 to router $R 5$, and the ATD between every two consecutive $S N D b$ packets at the router is inversely proportional to the capacity $C_{b}$ of narrow link $b$. In this example, we set $C_{a}=C_{b} / 2$, and thus the ATD between two consecutive SNDa packets is twice the ATD between two consecutive $S N D b$ packets as illustrated in Figure 5.

These 10 packets merge with one another at router $R 5$. In this example, we assume that these packets arrive at $R C V$ in the order of their arrival times at router $R 5$. For example,
Figure 5 shows that packet $b_{1}$ arrives at $R 5$ earlier than packet $a_{1}$, and thus packet $b_{1}$ arrives at $R C V$ earlier than packet $a_{1}$. In Section VI-C, we will discuss cases where this assumption does not hold and describe our solution. $R C V$ assigns the first received packet an arrival sequence number of 1 , and the next received packet an arrival sequence number of 2 , and so on. Figure 6 shows the arrival order of these 10 packets at $R C V$, and their corresponding arrival sequence numbers.
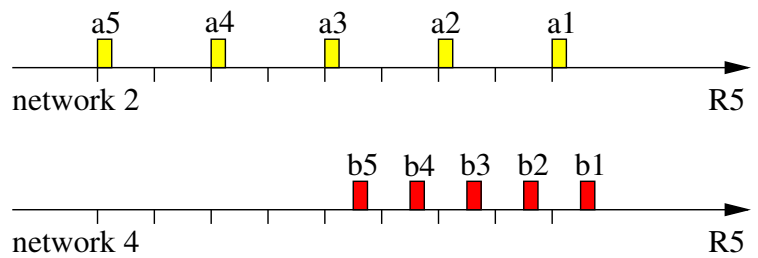

Fig. 5. $5 S N D a$ packets on the link from network 2 to router $R 5$ (top line), and at the same time $5 S N D b$ packets on the link from network 4 to $R 5$ (bottom line).

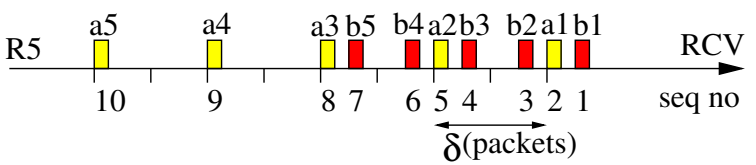

Fig. 6. The arrival sequence numbers of all 10 packets at $R C V$. The ASND $\delta$ between packets $a_{1}$ and $a_{2}$ is the difference between their packet arrival sequence numbers minus one.

The packet arrival sequence number difference (ASND, denoted by $\delta)$ of two packets is defined to be the difference between their arrival sequence numbers minus one. For example, the arrival sequence number of packet $a_{1}$ is 2 in Figure 6, and that of packet $a_{2}$ is 5 , thus their ASND is $\delta=(5-2)-1=2$ packets. Intuitively, this means that there are two other packets between packets $a_{1}$ and $a_{2}$.

2) ASND Histograms: We can infer the capacity ratio $\gamma$ of paths $a$ and $b$ by analyzing their ASND histograms. In this subsection, we present the concept of ASND histograms, and in Section $\mathrm{V}$, we will thoroughly study the impact of cross traffic on the ASND histograms.

Let $H_{a}(i)$ (respectively, $H_{b}(i)$ ) denote the total number of pairs of two consecutive packets that are sent by SNDa (respectively, $S N D b$ ) and separated by $\delta=i$ packets at $R C V$. For example, $H_{a}(0)=2$ pairs in Figure 6, because the ASND between packets $a_{3}$ and $a_{4}$ is 0 and that between packets $a_{4}$ and $a_{5}$ is also 0 . As another example, $H_{a}(2)=2$ pairs, because the ASND between packets $a_{1}$ and $a_{2}$ is 2 and that between packets $a_{2}$ and $a_{3}$ is also 2 .

The ASND histogram of the SNDa train is vector $H_{a}=$ $\left(H_{a}(0), H_{a}(1), H_{a}(2), \ldots\right)$, and that of the SNDb train is vector $H_{b}=\left(H_{b}(0), H_{b}(1), H_{b}(2), \ldots\right)$. For example, Figure 7 shows ASND histograms $H_{a}$ and $H_{b}$ for SNDa and SNDb trains, respectively.

We have the following theorem to simplify our analysis of ASND histograms.

Theorem 1: $\left|H_{a}(0)-H_{b}(0)\right| \leq 1$, if two trains have the same number $L$ of packets.

Proof: Let symbols $a$ and $b$ (without the subscripts) to 


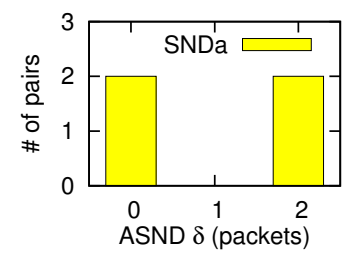

(a) Histogram $H_{a}$

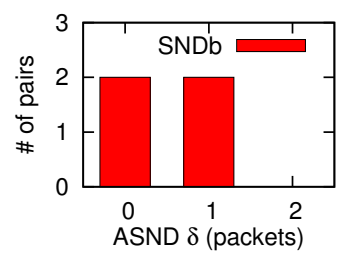

(b) Histogram $H_{b}$
Fig. 7. The ASND histograms of the two trains in Figure 6.

denote a packet of $S N D a$ and $S N D b$, respectively. The arrival order of the $2 L$ packets can be described by a string consisting of $L$ symbol $a$ 's and $L$ symbol $b$ 's. For example, if $L=5$, the arrival order of the 10 packets in Figure 6 can be described by string $a a a b b a b b a b$, where the rightmost symbol (i.e., b) is the first packet received by $R C V$ (i.e., $b_{1}$ ), and the leftmost symbol (i.e., $a$ ) is the last packet received by $R C V$ (i.e., $a_{5}$ ).

A string of $L$ symbol $a$ 's and $L$ symbol $b$ 's can be classified into the following four cases, according to the leftmost and rightmost symbols. We will prove only cases 1 and 2, and the other two cases can be proved very similarly.

- Case 1: The leftmost one: $a$, and the rightmost one: $a$.

- Case 2: The leftmost one: $a$, and the rightmost one: $b$.

- Case 3: The leftmost one: $b$, and the rightmost one: $a$.

- Case 4: The leftmost one: $b$, and the rightmost one: $b$.

Case 1: For a string with $2 L$ symbols, there are a total of $2 L-1$ pairs of two consecutive symbols. Let $n(a a), n(a b)$, $n(b a)$, and $n(b b)$ denote the number of pairs $a a, a b, b a$, and $b b$, respectively. Let $n(* a)$ and $n(* b)$ denote the number of pairs whose right symbol is $a$ and $b$, respectively. By definition, we have $n(* a)=n(b a)+n(a a)$, and $n(* b)=n(a b)+n(b b)$.

We have $n(* a)=L-1$, because the leftmost $a$ cannot be the right symbol of a pair. We also have $n(* b)=L$. Therefore, we have $n(* a)=n(* b)-1$.

Since both the leftmost and the rightmost symbols are $a$, we have $n(a b)=n(b a)$. Therefore, $H_{a}(0)=n(a a)=n(* a)-$ $n(b a)=(n(* b)-1)-n(a b)=n(b b)-1=H_{b}(0)-1$.

Case 2: We have $n(* a)=L-1$, because the leftmost $a$ cannot be the right symbol of a pair. We also have $n(* b)=L$. Therefore, we have $n(* a)=n(* b)-1$.

Since the leftmost symbol is $a$ and the rightmost one is $b$, we have $n(a b)=n(b a)+1$. Therefore, $H_{a}(0)=n(a a)=$ $n(* a)-n(b a)=(n(* b)-1)-(n(a b)-1)=n(b b)=H_{b}(0)$.

For example, $H_{a}(0)-H_{b}(0)=2-2=0$ in Figure 7. Note that, Theorem 1 holds no matter whether there is cross traffic or not and no matter how long the train size $L$ is.

We will not show and will not use $H_{a}(0)$ and $H_{b}(0)$ in the rest of the paper for the following two reasons. First, usually the $S N D a$ and $S N D b$ trains only partially overlap with each other, and thus $H_{a}(0)$ and $H_{b}(0)$ are mainly due to the non-overlapping packets of the two trains. Second, Theorem 1 shows that $H_{a}(0)$ and $H_{b}(0)$ are very close to each other, and thus do not provide much useful information.

A peak (also called a mode) in a histogram is a local maximum that is higher than its right neighbors and no less than its left neighbor (if exists). For example, histogram $H_{a}$ in Figure 7 has a peak at 2 packets, and histogram $H_{b}$ has a peak at 1 packet (note that it does not have the left neighbor, since we do not consider $\left.H_{b}(0)\right)$.

We introduce the second theorem about the peaks in ASND histograms. Without loss of generality, this theorem considers only case $C_{a} \leq C_{b}$.

Theorem 2: In the absence of cross traffic, if $C_{a} \leq C_{b}$, histogram $H_{a}$ has only one peak and the peak is located at $\Gamma$ packets, and histogram $H_{b}$ has only one peak and the peak is located at 1 packet. The capacity ratio $\gamma$ can be obtained as follow, where $H_{a}(\Gamma-1)$ should be set to 0 if $\Gamma=1$.

$$
\gamma=\frac{(\Gamma-1) H_{a}(\Gamma-1)+\Gamma H_{a}(\Gamma)+(\Gamma+1) H_{a}(\Gamma+1)}{H_{a}(\Gamma-1)+H_{a}(\Gamma)+H_{a}(\Gamma+1)}
$$

Proof: When there is no cross traffic, the ATD of a pair of two consecutive $S N D a$ packets is $S / C_{a}$. The average number of $S N D b$ packets that can be transmitted during an $S / C_{a}$ interval is $\left(S / C_{a}\right) \times\left(C_{b} / S\right)=C_{b} / C_{a}$. Therefore, the average number of $S N D b$ packets between a pair of two consecutive $S N D a$ packets is $C_{b} / C_{a}$. We consider the following three possible cases:

Case 1: $C_{b} / C_{a}$ is a positive integer. That is, $\Gamma=\gamma=$ $C_{b} / C_{a}$. In this case, there are exactly $\Gamma S N D b$ packets between a pair of two consecutive $S N D a$ packets. Therefore, the peak of $H_{a}$ is at $\Gamma$ packets. In this case, there are either 0 or 1 $S N D a$ packet between a pair of two consecutive $S N D b$ packets. Therefore, the peak of $H_{b}$ is at 1 packet. Note that, $H_{a}(\Gamma-$ 1) $=H_{a}(\Gamma+1)=0$, and thus Equation (4) can be proved.

Case 2: $C_{b} / C_{a}$ is a decimal greater than 1 , and $\Gamma=$ $\left\lfloor C_{b} / C_{a}\right\rfloor$ and $\Gamma+1=\left\lceil C_{b} / C_{a}\right\rceil$. In this case, there are either $\left\lfloor C_{b} / C_{a}\right\rfloor$ or $\left\lceil C_{b} / C_{a}\right\rceil S N D b$ packets between a pair of two consecutive SNDa packets. Because $\Gamma=\operatorname{round}(\gamma)=$ $\left\lfloor C_{b} / C_{a}\right\rfloor$, we have $H_{a}(\Gamma)>H_{a}(\Gamma+1)>0$. Therefore, the peak of $H_{a}$ is at $\Gamma$ packets. In this case, there are either 0 or $1 S N D a$ packet between a pair of two consecutive $S N D b$ packets. Therefore, the peak of $H_{b}$ is at 1 packet. Note that, $H_{a}(\Gamma-1)=0$, and thus Equation (4) can be proved.

Case 3: $C_{b} / C_{a}$ is a decimal greater than 1 , and $\Gamma-1=$ $\left\lfloor C_{b} / C_{a}\right\rfloor$ and $\Gamma=\left\lceil C_{b} / C_{a}\right\rceil$. This case can be proved in a similar way to case 2 .

For example, in Figure 7, because $C_{a}<C_{b}$, histogram $H_{a}$ has a peak at $\Gamma=\operatorname{round}\left(C_{b} / C_{a}\right)=2$ packets, and histogram $H_{b}$ has a peak at 1 packet.

3) ASND-based Capacity Comparison: Theorem 2 provides the foundation of our proposed capacity comparison method in the absence of cross traffic. Given the histogram $H$ of the slower path, algorithm EST-RATIO can estimate the capacity ratio $\gamma$ using Theorem 2 . Since initially we do not know which path is slower, algorithm COMPARE calculates two ratio estimates: $\gamma_{a}$ assuming path $a$ is slower, and $\gamma_{b}$ assuming path $b$ is slower. Then it selects the bigger ratio as the final result. 

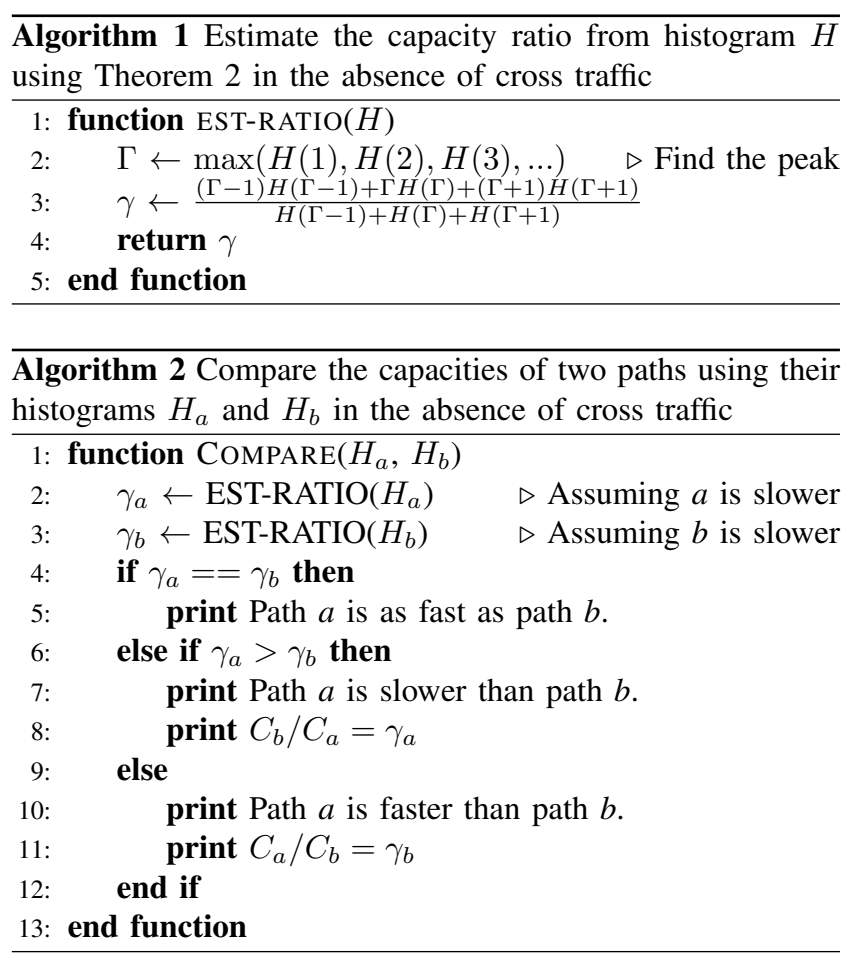

\section{IMPACT OF CROSS TRAFFIC}

In this section, we study the impact of various types of cross traffic on the ASND histograms using our lab testbed.

We study five possible types of cross traffic as illustrated in Figure 8, which is very similar to Figure 3 and just simplifies each network to a single router. The capacity of each link is chosen to demonstrate the impact of the cross traffic on that link. We emulate this network using our 10Gbps testbed, and each link is emulated by a Linux token bucket filter (tbf).

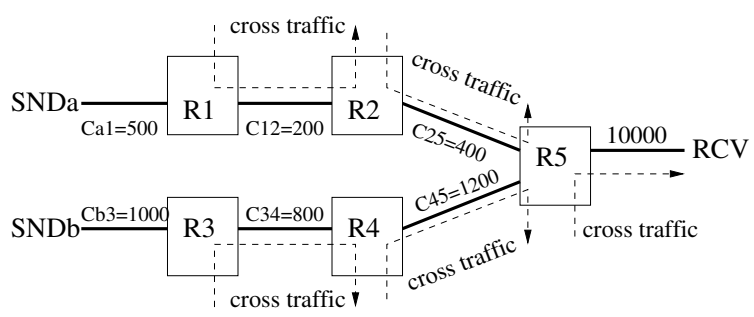

Fig. 8. Five possible sources of cross traffic. Link capacity unit: Mbps.

The narrow link of path $a$ from $S N D a$ to $R C V$ is the link between routers $R l$ and $R 2$, and thus the capacity of path $a$ is $C_{a}=200 \mathrm{Mbps}$. The narrow link of path $b$ from $S N D b$ to $R C V$ is the link between routers $R 3$ and $R 4$, and thus the capacity of path $b$ is $C_{b}=800 \mathrm{Mbps}$. Therefore, we have $\Gamma=\gamma=4$.

In each of the following experiments, each sender sends a train of $L=500$ packets at approximately the same time, and $R C V$ measures the ASND histograms. Because path $b$ is the faster one, all $S N D b$ histograms concentrate at 0 and 1 packet (similar to Figure 7(b)), and thus we do not show the $S N D b$

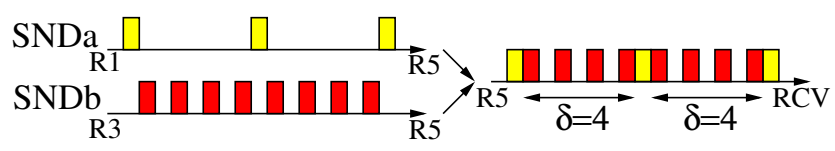

Fig. 9. No cross traffic. Each box indicates a packet on the link, but the width of a box does not represent its transmission time.

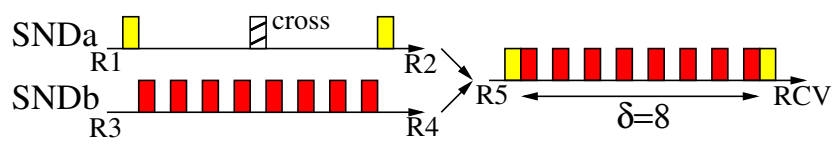

Fig. 10. Cross traffic between $R 1$ and $R 2$.

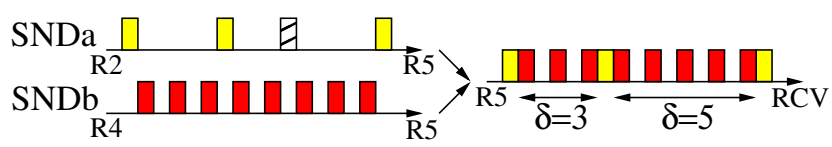

Fig. 11. Cross traffic between $R 2$ and $R 5$.

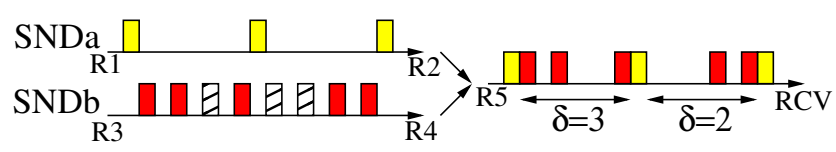

Fig. 12. Cross traffic between $R 3$ and $R 4$.

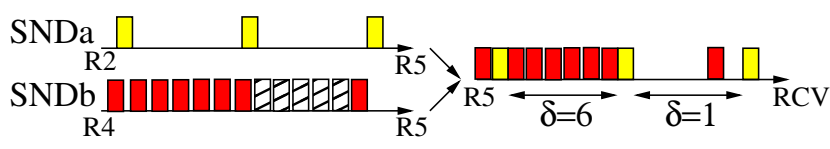

Fig. 13. Cross traffic between $R 4$ and $R 5$.

histograms. For the SNDa histograms, we do not show the result for 0 packet, as explained in Section IV.

1) No Cross Traffic: As a reference case, first we do not generate any cross traffic. Since $\gamma=4$, there should be $4 S N D b$ packets between a pair of two consecutive SNDa packets, as illustrated in Figure 9. The SNDa histogram is shown in Figure 14. As we expect, the ASND of most SNDa pairs is 4 packets. But there are a small number of $S N D a$ pairs with other ASNDs, which are mainly caused by the randomness of the routers that are emulated using our lab computers and Linux tbf.

2) Cross Traffic between R1 and R2: This experiment shows the impact of cross traffic before or on the narrow link of path $a$ (i.e., the slower path). Random cross traffic is generated using MGEN [15] at an average rate of $200 * 50 \%=100$ Mbps between $R 1$ and $R 2$.

Let's consider the example shown in Figure 10. There are still the same $8 S N D b$ packets passing the link between $R 3$ and $R 4$ as in Figure 9. But during this time interval, a cross traffic packet is inserted between the first (i.e., the rightmost one) and second SNDa packets (the third SNDa packet is further delayed, and not shown in the figure). As a result, the ASND between the first and second SNDa packets is doubled and becomes 8 packets.

This is why the SNDa histogram in Figure 15 has a nonnegligible number of $S N D a$ pairs with $\delta=8$ packets. Further more, the numbers of SNDa pairs with $\delta=\gamma i=4 i$ packets approximately follow a Geometric distribution described by Equation (5), where $N$ is the total number of pairs with $\delta=4 i$ packets, and $p$ is the occurrence probability of a cross traffic 


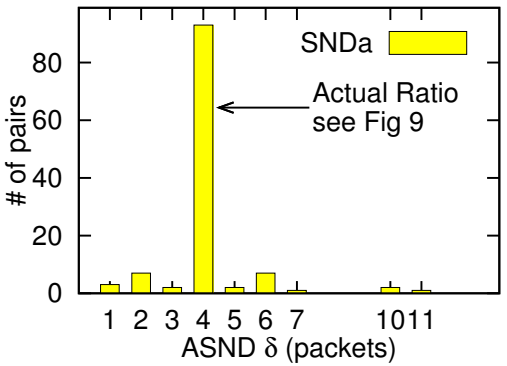

Fig. 14. No cross traffic.

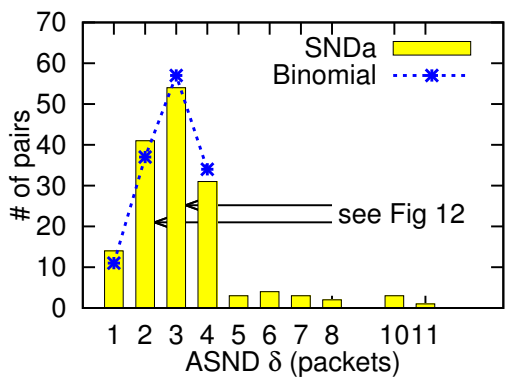

Fig. 17. $50 \%$ cross traffic between $R 3$ and $R 4$.

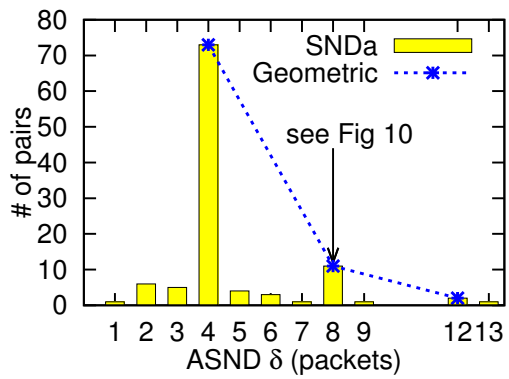

Fig. 15. $50 \%$ cross traffic between $R 1$ and $R 2$

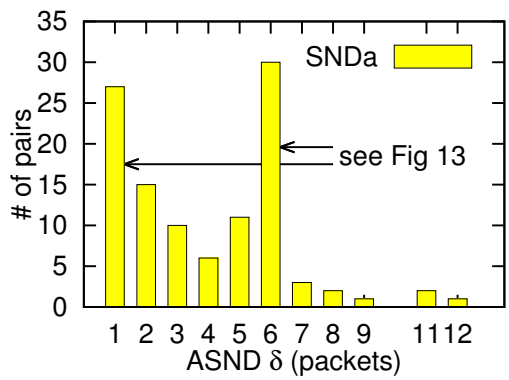

Fig. 18. $50 \%$ cross traffic between $R 4$ and $R 5$.

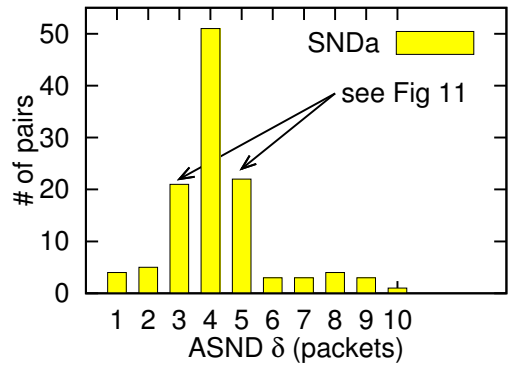

Fig. 16. $80 \%$ cross traffic between $R 2$ and $R 5$.

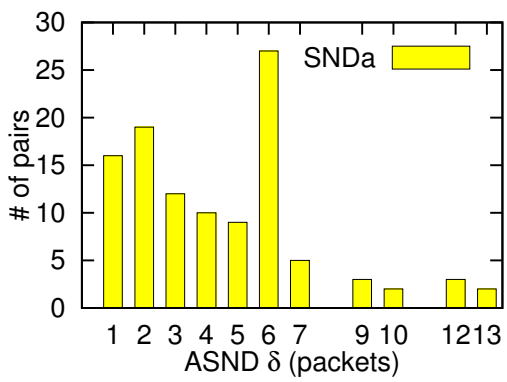

Fig. 19. $50 \%$ cross traffic on all 5 links. packet. For example, the dotted line in Figure 15 is obtained using Equation (5) with the corresponding $N$ and $p$.

$$
H_{a}(i * 4)=N p^{i-1}(1-p) \quad 1 \leq i
$$

3) Cross Traffic between R2 and R5: This experiment shows the impact of cross traffic beyond the narrow link of path $a$ but still before the shared segment. Random cross traffic is generated at an average rate of $400 * 80 \%=320 \mathrm{Mbps}$ between $R 2$ and $R 5$.

In the example shown in Figure 11, there are still the same $8 S N D b$ packets passing the link between $R 4$ and $R 5$ as in Figure 9. But a cross traffic packet is inserted between the first and second $S N D a$ packets. Because the link capacity between $R 2$ and $R 5$ is twice that between $R 1$ and $R 2$, the third SNDa packet can still be transmitted at the original time as in Figure 9. As a result, the ASND between the first and second SNDa packets increases to 5 packets, but the ASND between the next two SNDa packets decreases to 3 packets.

This is why the SNDa histogram shown in Figure 16 has a non-negligible number of SNDa pairs with ASNDs around 4 packets, such as 3 and 5 packets.

4) Cross Traffic between R3 and R4: This experiment shows the impact of cross traffic before or on the narrow link of path $b$ (i.e., the faster path). Random cross traffic is generated at an average rate of $800 * 50 \%=400 \mathrm{Mbps}$ between $R 3$ and $R 4$.

In the example shown in Figure 12, there are still the same $3 S N D a$ packets passing the link between $R 1$ and $R 2$ as in Figure 9. But three cross traffic packets are inserted between these $S N D b$ packets (the rightmost three $S N D b$ packets in Figure 9 are further delayed, and not shown in Figure 12). As a result, the ASND between the first and second SNDa packets decreases to 2 packets, and the ASND between the next two $S N D a$ packets decreases to 3 packets.

This is why the SNDa histogram in Figure 17 has a large number of SNDa pairs with ASND less than 4 packets. The numbers of SNDa pairs with ASNDs between 1 and 4 packets follow a Binomial distribution described by Equation (6), where $N$ is the total number of SNDa pairs with ASNDs between 1 and 4 packets, and $p$ is the occurrence probability of a cross traffic packet. The dotted line in Figure 17 is obtained using Equation (6) with the corresponding $N$ and $p$.

$$
H_{a}(i)=N\left(\begin{array}{l}
4 \\
i
\end{array}\right)(1-p)^{i} p^{4-i} /\left(1-p^{4}\right) \quad 1 \leq i \leq 4
$$

5) Cross Traffic between R4 and R5: This experiment studies the impact of cross traffic beyond the narrow link of path $b$ but still before the shared segment. Random cross traffic is generated at an average rate of $1200 * 50 \%=600 \mathrm{Mbps}$ between $R 4$ and $R 5$.

In the example shown in Figure 13, there are still the same 3 SND $a$ packets passing the link between $R 2$ and $R 5$ as in Figure 9. But during this time interval, several cross traffic packets are inserted between the first and second $S N D b$ packets. Because the link capacity between $R 4$ and $R 5$ is higher than that between $R 3$ and $R 4$, the remaining SNDa packets are only slightly delayed than in Figure 9. As a result, the ASND between the first and second SNDa packets decreases to 1 packet, and the ASND between the next two $S N D a$ packets becomes 6 packets, which is the capacity ratio of the link between $R 4$ and $R 5$ to the link between $R 1$ and $R 2$ (i.e., $1200 / 200=6$ ).

This is why the SNDa histogram shown in Figure 18 has a large number of SNDa pairs with ASND not equal to 4 packets, such as 1 and 6 packets. 
6) Cross Traffic between $\mathrm{R} 5$ and $\mathrm{RCV}$ : This experiment shows the impact of cross traffic in the shared segment of both paths. As we expect, this type of cross traffic does not have any impact on the histograms.

7) Cross Traffic on All Five Links: Finally, we generate all five types of cross traffic. The SNDa histogram is shown in Figure 19, and it shows the combination of the impact of all five types of cross traffic.

8) Summary: We have the following observations about the ASND histogram of the slower path.

Observation 1: In practice, the ASND histogram could have a small number of ASND values caused by the randomness of the end-systems and the networks. These ASND values should be treated as noises and be discarded.

Observation 2: With little or no cross traffic, there is only one peak and the peak is located at the rounded capacity ratio $\Gamma$ (e.g., Figure 14). This is consistent with Theorem 2.

Observation 3: In the presence of cross traffic, it is possible that there are multiple peaks (also called multi-mode), and $\Gamma$ may or may not be the location of a peak. For example, Figure 18 has peaks at 1 and 6 packets, but no peak at 4 packets. We observe that multiple peaks are usually caused by the cross traffic on the faster path. More specifically, they are usually caused by the cross traffic beyond the narrow link of the faster path, e.g., in Figures 18 and 19.

Observation 4: In the presence of cross traffic, if there is only one peak, the peak location tends to be a lower bound of $\Gamma$ (e.g., Figure 16 has a single peak at 4 packets, and Figure 17 has a single peak at 3 packets). Intuitively, this is because an ASND value greater than $\Gamma$ usually leads to another ASND value smaller than $\Gamma$ (e.g., Figures 11 and 13). Therefore, if there is a peak to the right of $\Gamma$, then there is usually another peak to the left of $\Gamma$. That is, there will be multiple peaks.

Observation 5: We have calculated and verified that the average of all ASND values of a histogram could be higher than or lower than $\gamma$ (i.e., neither an upper bound nor a lower bound), depending on the amounts and locations of cross traffic on both paths.

\section{PATHCOMP}

In this section, we present our proposed PathComp to relative compare the capacity ratio of two paths to the same receiver without requiring accurate packet time information.

\section{A. The PathComp Method}

PathComp follows the basic idea of algorithms EST-RATIO and COMPARE as described in Section IV-C3. However, there are two problems with algorithm EST-RATIO in the presence of cross traffic. 1) It is possible to have multiple peaks in a histogram mainly due to the cross traffic on the faster path (i.e., Observation 3 in Section V-8), however EST-RATIO assumes only one peak in a histogram. To tackle this problem, we divide the long packet train on the faster path into multiple short packet blocks, in order to reduce the impact of cross traffic. 2) If there is a single peak in the histogram of the slower path, the peak location tends to be a lower bound of $\Gamma$ (i.e.,

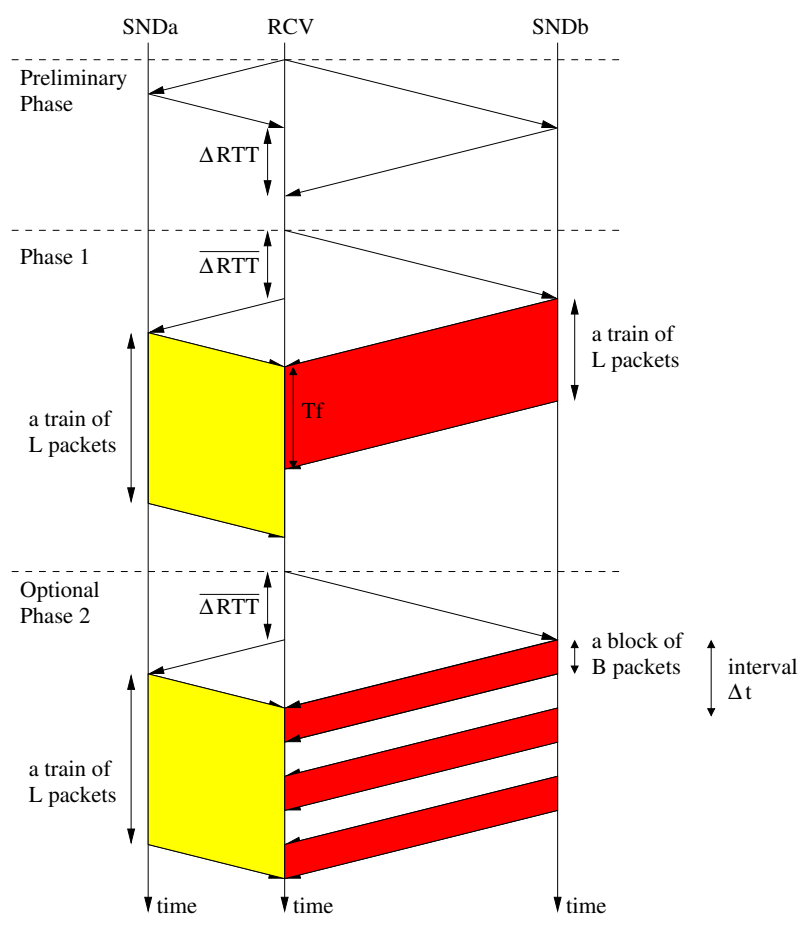

Fig. 20. PathComp has three phrases.

Observation 4 in Section V-8). To tackle this problem, we estimate $\Gamma$ by the peak of the weighted histogram.

PathComp consists of three phases as shown in Figure 20.

- 1) Preliminary phase measures some basic network information, such as the round-trip times (RTTs).

- 2) Phase I measures the histograms of the two paths. If there is a single peak in the histogram of the slower path, PathComp estimates $\gamma$ using algorithms COMPARE and EST-RATIO2; otherwise, it starts Phase II.

- 3) Phase II re-measures the histograms using multiple packet blocks on the faster path, and then estimates $\gamma$ using algorithm EST-RATIO2.

Figure 20 still considers the two paths shown in Figure 3. But to simplify the figure, Figure 20 assumes that there is only one link between $S N D a$ and $R 5$ that is the narrow link of path $a$, and there is only one link between $S N D b$ and $R 5$ that is the narrow link of path $b$.

1) Preliminary Phase: This phase measures the RTT difference $\triangle R T T$ between $S N D a-R C V$ and $S N D b-R C V$ as illustrated in Figure 20, so that in the next two phases the packets of $S N D a$ and $S N D b$ can overlap with each other. PathComp measures $\triangle R T T$ multiple times, and calculates the mean (denoted by $\overline{\triangle R T T}$ ) and standard deviation (denoted by $\sigma(\triangle R T T)$ ) of measured $\triangle R T T$ values.

2) Phase I: $R C V$ first tells the sender with a longer RTT (i.e., $S N D b$ in Figure 20) to start its packet transmission, and after a delay of $\overline{\triangle R T T}$, RCV then tells the sender with a shorter RTT (i.e., SNDa) to start its packet transmission. Each sender sends a train of $L$ consecutive packets with the same packet size $S$. In Figure 20, the capacity $C_{a}$ of path $a$ is lower 
than the capacity $C_{b}$ of path $b$, so $S N D a$ takes a longer time to transmit the same number $L$ of packets than $S N D b$.

After $R C V$ receives these two trains, PathComp measures ASND histograms $H_{a}$ and $H_{b}$ of the two paths, and uses Algorithms EST-RATIO2 and COMPARE2 to estimate the capacity ratio. The difference between EST-RATIO and EST-RATIO2 is that the former selects the peak from the original histogram $H=(H(1), H(2), H(3), \ldots)$, whereas the latter selects the peak from the weighted histogram $(H(1), 2 H(2), 3 H(3), \ldots)$. This is motivated by Observation 4 in Section V-8. Note that the peak location of the weighted histogram is greater than or the same as that of the original histogram. The difference between COMPARE and COMPARE2 is that the former calls EST-RATIO whereas the latter called EST-RATO2. In addition, if multiple peaks are detected in the histogram of the slower path, Algorithm COMPARE2 starts phase II.
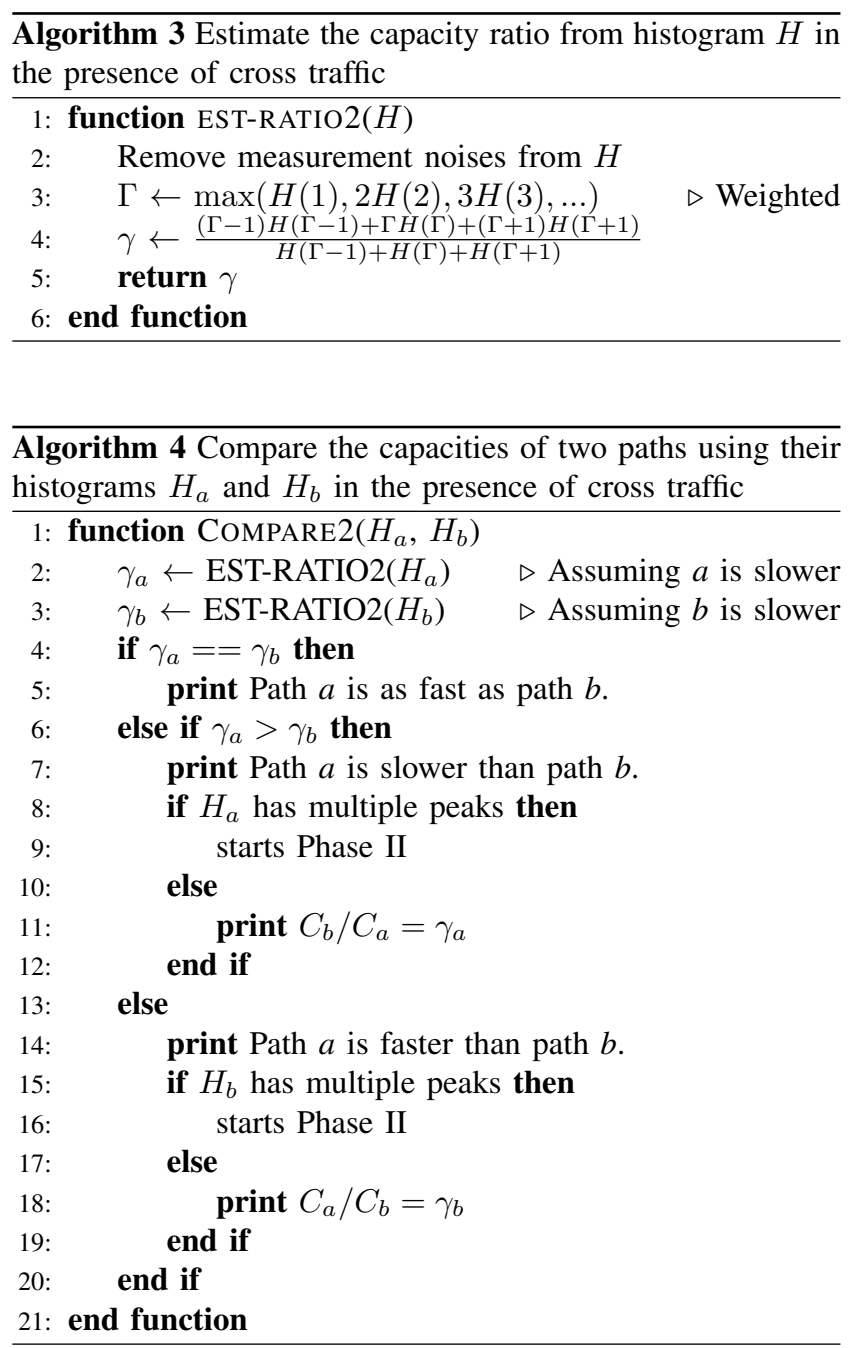

Parameter Setting: If $\sigma(\triangle R T T)=0$, the two trains should arrive at $R C V$ at the same time as illustrated in Figure 20. In practice, $\sigma(\triangle R T T)>0$, and the train size $L$ should be sufficiently long so that the two trains can still overlap with each other. For example, consider a cloud computing network in a data center with $\sigma(\triangle R T T)=1 \mathrm{~ms}$ and with the capacity=1 Gbps, $L$ should be at least 83 packets longer to compensate for the RTT variance if packet size $S$ is 1500 Byte. By default, PathComp sets the train size $L$ to 500 packets.

If the two trains could not overlap with each other, or overlap for only a small portion of each train, PathComp increases the train size and re-sends the two trains. However, if excessive packet loss is detected at $R C V$, PathComp quits the estimation.

By default, PathComp sets the packet size $S$ to 1500 bytes. This is because our experiments show that ASND histograms become hard to predict and analyze when the packet size is small. Intuitively, this is because the randomness of the endsystems and networks have a big impact on small packets, and thus there are much more noises in the ASND histograms.

3) Phase II: PathComp enters this phase, if there are multiple peaks in the histogram of the slower path. As observed in Section V-8, this is usually due to the high cross traffic load on the faster path. Therefore, we divide the long packet train on the faster path into multiple short packet blocks, in order to reduce the impact of cross traffic.

Specifically, PathComp still sends a train of $L$ packets backto-back on the slower path. But on the faster path, PathComp sends a block of $B$ packets back-to-back every $\Delta t$ time interval, until all $L$ packets have been sent out, as illustrated in Figure 20. After $R C V$ receives these two trains, PathComp measures only the ASND histogram of the slower path, and uses Algorithm EST-RATIO2 to estimate the capacity ratio.

Parameter Setting: The block size $B$ should be much larger than the capacity ratio $\gamma$, because $B$ limits the maximum ASND between two consecutive packets on the slower path. By default, PathComp sets $B$ to 20 packets, which is larger than most typical ratios, such as 2 and 10 .

The interval $\Delta t$ should be long enough in order to sufficiently separate different packet blocks, but should not be too long so that most packets on the faster path can still overlap with the packets on the slower path. By default, PathComp sets $\Delta t$ to $2 T_{f} /(L / B)=2 B T_{f} / L$, so that the average transmission rate of all packets is approximately reduced by half and the total transmission time of all packets is approximately doubled. $T_{f}$ is the time for $R C V$ to receive the packet train from the faster path in Phase I.

PathComp checks whether $\Delta t$ is too long or too short as follows. If less than half of the packet blocks on the faster path overlap with the packet train on the slower path, it is likely that $\Delta t$ is too big. If the ASND histogram of the faster path contains very few large ASND values (e.g. $\delta \geq 5$ ), it is likely that $\Delta t$ is too short. In these cases, PathComp adjusts the interval $\Delta t$ and re-sends the packets.

As an example, Figure 19 shows the original SNDa histogram with multiple peaks obtained using the packet train on the faster path, and Figure 21 shows the new SNDa histogram obtained using packet blocks on the faster path. We can see that in the new histogram, there are still multiple peaks, but there is a peak at $\Gamma=4$, and it is the highest peak. 


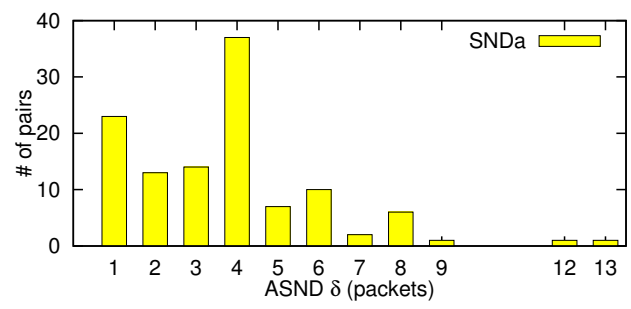

Fig. 21. The difference between this figure and Figure 19 shows the effectiveness of Phase II in the presence of cross traffic.

\section{B. Packet Time Information used in PathComp}

PathComp uses only two types of coarse packet time information: the $\triangle R T T$ between two paths, and the time $T_{f}$ for $R C V$ to receive the packet train from the faster path in Phase I. None of them needs to be accurately measured.

$\triangle R T T$ is used in both Phases I and II so that the packets on both paths will arrive at $R C V$ at approximately the same time. The inaccuracy in measuring $\triangle R T T$ can be mitigated by using longer packet trains.

$T_{f}$ is used in Phase II to calculate block interval $\Delta t$. Note that time $T_{f}$ is the time for receiving a train of $L$ packets, not a single packet. Therefore, due to the relatively large value of $L$ (e.g., 500), $T_{f}$ is a relatively long time (e.g., $0.6 \mathrm{~ms}$ at $10 \mathrm{Gbps})$. In addition, too large or too small interval $\Delta t$ due to the inaccuracy in measuring $T_{f}$ will be detected and adjusted by PathComp in Phase II.

\section{An Implementation Challenge: RSS and IC}

Two features of high-speed NICs may interfere with PathComp: Receiver Side Scaling (RSS) and Interrupt Coalescence (IC). Each of them alone doe not affect PathComp, but when both of them are enabled, they greatly interfere with PathComp. Below we explain the reasons and our solution.

RSS [14] is a relatively new NIC feature to allow a NIC to balance interrupts among multiple CPUs in a computer. RSS distributes incoming packets into different NIC Rx queues according to their hash values calculated using the packet information, such as source IP. As a result, the probing packets from two different senders are placed into different NIC Rx queues and handled by different CPUs on $R C V$. IC [9] is a NIC feature to reduce the CPU load by generating an interrupt for a group of packets instead of each packet.

When an interrupt is generated as each packet arrives (i.e., IC disabled), RSS alone does not affect PathComp because the interrupt sequence follows the packet arrival sequence. When there is only a single NIC Rx queue (i.e., RSS disabled), IC along does not affect PathComp because IC changes only the packet arrival times but not the packet arrival sequence. However, when both RSS and IC are enabled, they greatly interfere with PathComp as illustrated in Figure 22. Packets from different senders are placed into different NIC Rx queues, and an interrupt is generated only for a group of packets from a Rx queue. As a result, the packet arrival sequence measured by PathComp is different from the original packet arrival sequence at the NIC.

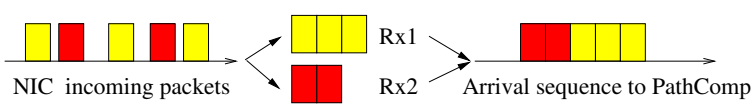

Fig. 22. Impact of RSS and IC on the packet arrival sequence.

A simple solution is IP address spoofing. We modify the packet source IP address of one sender to the same as that of the other sender, in order to conceal $R C V$ that all packets are from the same sender. $R C V$ therefore places all packets to the same Rx queue. Although packets with a forged source IP address may be filtered by some firewall, this is a more practical solution compared with disabling either RSS or IC on $R C V$. We have successfully tested this solution on our campus network, Amazon EC2 [1], and PlanetLab [18].

Figure 23 shows the SNDa histogram when both RSS and IC are enabled. It is obtained with exactly the same testbed setting (including cross traffic, RSS, and IC) as Figure 14, except that the latter uses IP address spoofing. We can see that Figure 23 is greatly different from Figure 14. That is, without IP address spoofing, RSS and IC greatly change the packet arrival sequence.

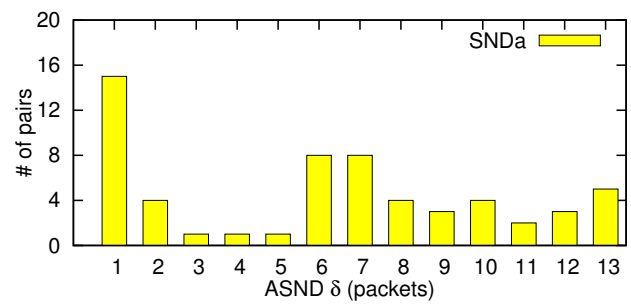

Fig. 23. The difference between this figure and Figure 14 shows the impact of RSS and IC on the histogram.

\section{EVALUATION}

In this section, we evaluate PathComp using our lab testbed, our campus network, and Amazon EC2.

\section{A. Testbed Results}

We conduct the following three groups of testbed experiments to evaluate PathComp with default parameters. For each experiment, we run it for 50 times, and report the average with a $95 \%$ confidence interval. The emulated network topology is the same as the one shown in Figure 8 but with different link capacities. We use Linux tbf with the minimum token burst size to emulate a link capacity, except $100 \mathrm{Mbps}, 1 \mathrm{Gbps}$, and 10 Gbps. We notice that Linux tbf on our testbed can only emulate up to $1.6 \mathrm{Gbps}$ links due to limited system capability. Thus, the maximum link capacity in our testbed experiments is $1.6 \mathrm{Gbps}$, except $10 \mathrm{Gbps}$.

Group 1 - Impact of Large Capacity Ratios: This group of experiments study the accuracy of PathComp when two paths have a capacity ratio at least 2. For path $a$ in Figure 8, we set $C_{a 1}=500 \mathrm{Mbps}, C_{12}=200 \mathrm{Mbps}, C_{25}=1 \mathrm{Gbps}$, and thus the capacity of path $a$ is $C_{a}=C_{12}=200 \mathrm{Mbps}$. For path $b$, we set $C_{b 3}=1.6 \mathrm{Gbps}, C_{34}=400 \mathrm{Mbps}$ to $1.6 \mathrm{Gbps}$, 
$C_{45}=10 \mathrm{Gbps}$, and thus the capacity of path $b$ is $C_{b}=C_{34}$ Therefore, the capacity ratio $\gamma=C_{b} / C_{a}$ varies from 2 to 8 .

The estimated capacity ratios are shown in Figure 24(a), where each link marked in Figure 8 has $30 \%$ cross traffic. We can see that PathComp can accurately measure these large capacity ratios. The large confidence interval at $\gamma=8$ is partially because that Linux tbf has almost reached its max performance limit on our testbed.

Group 2 - Impact of Small Capacity Ratios: This group of experiments study the accuracy of PathComp when two paths have a capacity ratio no more than 2 . Path $a$ has the same link capacities as in group 1 , and thus the capacity of path $a$ is still $C_{a}=C_{12}=200 \mathrm{Mbps}$. For path $b$, we set $C_{b 3}=1 \mathrm{Gbps}$, $C_{34}=200 \mathrm{Mbps}$ to $400 \mathrm{Mbps}, C_{45}=1 \mathrm{Gbps}$, and thus the capacity of path $b$ is $C_{b}=C_{34}$. Therefore, the capacity ratio $\gamma=C_{b} / C_{a}$ varies from 1 to 2 .

The estimated capacity ratios are shown in Figure 24(b), where each link marked in Figure 8 has $30 \%$ cross traffic. We can see that PathComp can accurately measure these small capacity ratios. Even when $\gamma=2$, the average estimated ratio is 1.88 , and is very close to the actual ratio. Note that results with $\gamma=2$ in Figures 24(a) and 24(b) are obtained using different link capacities (e.g., $C_{45}$ ) and then different amounts of cross traffic. In the latter, $C_{45}$ is smaller, and thus its link is more congested. This is why the estimation error with $\gamma=2$ in the latter is larger than that in the former.

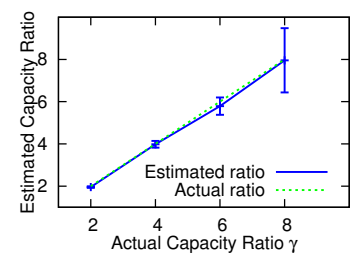

(a) Capacity ratio in range $(2,8)$

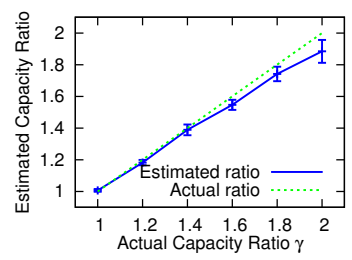

(b) Capacity ratio in range $(1,2)$
Fig. 24. Impact of large and small capacity ratios.

Group 3 - Impact of Cross Traffic: This group of experiments study the accuracy of PathComp under different amounts of cross traffic. We use the same link capacities as in group 2, except that we set $C_{34}$ to $400 \mathrm{Mbps}$. Therefore, $\gamma=C_{b} / C_{a}$ is fixed to 2 .

Figure 25(a) shows the estimated capacity ratios when the cross traffic on path $a$ varies from $10 \%$ to $60 \%$ and that on path $b$ is fixed to $30 \%$. Figure 25 (b) shows the estimated capacity ratios when the cross traffic on path $a$ is fixed to $30 \%$, and that on path $b$ varies from $10 \%$ to $60 \%$.

We can see that cross traffic on path $b$ (i.e., the faster path) has a bigger impact than that on past $a$ (i.e., the slower path). The reason is the probing traffic on path $b$ is sent at a higher rate. With the same percentage of crossing traffic, path $b$ is more congested than path $a$. For example, with $60 \%$ crossing traffic, the link utilization between $R 4$ and $R 5$ on path $b$ can reach up to $0.6+400 / 1000=100 \%$, but only up to $0.6+$ $200 / 1000=80 \%$ for the link between $R 2$ and $R 5$ on path $a$. This is consistent with our observation in Section $\mathrm{V}$, and this is also the motivation why PathComp in Phase II divides a long packet train into multiple short packet blocks on the faster path.

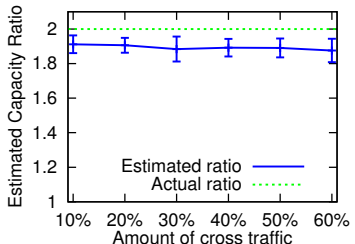

(a) Varying cross traffic on path $a$

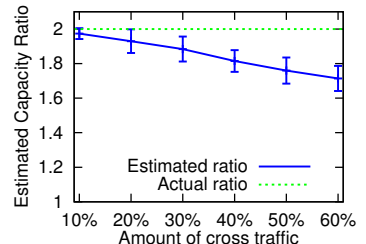

(b) Varying cross traffic on path $b$
Fig. 25. Impact of cross traffic.

Remarks: We also run PathRate [6] on our testbed, which is one of the most well studied and widely used capacity estimation methods. However, it could not accurately estimate the capacity of a path on our testbed. For example, in Group 1 , it reports a capacity of $1100 \sim 1400 \mathrm{Mbps}$ (results of multiple runs) for path $a$, and reports an insufficient number of packet dispersion estimates for path $b$. This is partially due to interference of IC and Linux tbf.

\section{B. Campus Network Results}

We also evaluate PathComp using some servers in our campus network, where we know the network and server information.

Intra-Department Network: We choose three servers, denoted by $S N D a, S N D b$, and $R C V$, in our department. SNDa is connected to the department 1 Gbps network through a 100 Mbps switch, and both $S N D b$ and $R C V$ are connected to the department network through 1 Gbps Ethernet. Figure 26(a) shows the ASND histograms of SNDa and $S N D b$, and note that there are some ASND values at 9 and 11 packets which are caused by cross traffic. PathComp correctly estimates that the capacity ratio is 10 (corresponding to the peak at $\delta=10$ packets). We also run PathRate, and it correctly estimates the capacity between $S N D a$ and $R C V$ as $100 \mathrm{Mbps}$, but it mistakenly reports the capacity between $S N D b$ and $R C V$ as 1900 2100 Mbps.

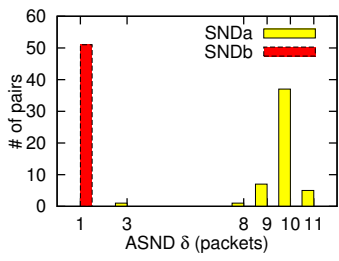

(a) Intra-Department Network

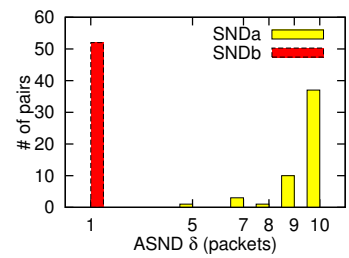

(b) Inter-Department Network
Fig. 26. Campus network experiments.

Inter-Department Network: We choose three servers, denoted by $S N D a, S N D b$, and $R C V$, in three different departments in our campus network. SNDa has a 100 Mbps NIC, and both $S N D b$ and $R C V$ have a 1 Gbps NIC. All three servers are connected to the campus 1 Gbps network. Each of the two paths passes four routers, and they share only the last router 
just before $R C V$. Figure 26(b) shows the ASND histograms of $S N D a$ and $S N D b$, and PathComp correctly estimates that the capacity ratio is 10 . We also run PathRate, and it correctly estimates the capacities of both paths: SNDa: $100 \mathrm{Mbps}$, and SNDb: 970 990 Mbps.

\section{Amazon EC2 Results}

We also evaluate PathComp using VMs on Amazon Elastic Compute Cloud (EC2) [1], which is a very popular public cloud computing platform. The EC2 facilities are located at multiple locations, and we choose the one in the US West (Oregon) region that includes three zones. We select three micro instances from different zones as three senders denoted by $S N D a, S N D b$, and $S N D c$, and we select one medium instance as the receiver $R C V$.

We relatively compare the path capacities from the three senders to the receiver for 100 times, and Figure 27 shows the cumulative distribution function (CDF) of the estimated capacity ratios. PathComp reports that $S N D a$ is slightly faster than $S N D b, S N D b$ is about 2.2 2.4 times faster than $S N D c$, and $S N D a$ is about 2.4 2.7 times faster than $S N D c$. We can also see that the results are highly consistent. For example, among estimated ratios between $S N D c$ and $S N D a$, most of them are about $2.4 \sim 2.7$, and about $10 \%$ of them are smaller than 2.4. This is possibly due to the interference of VM scheduling, as micro instances are scheduled much more frequently than other types of instances.

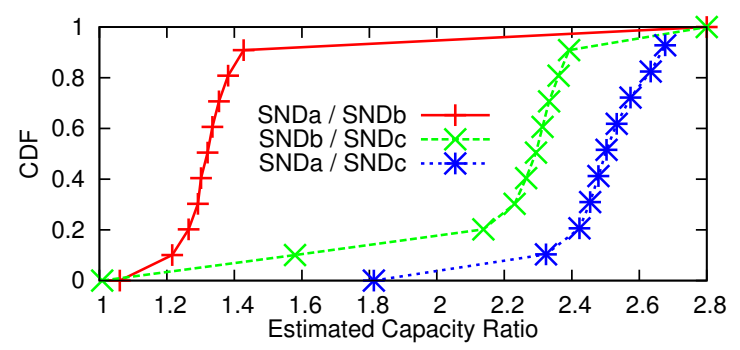

Fig. 27. Amazon EC2 Results.

In order to verify our estimated capacity ratios, we also run PathRate and iperf on EC2. PathRate reports that IC is detected and there is an insufficient number of packet dispersion estimates. Since this paper considers the capacity of a path that indicates the short-term peak rate of the path, we use the iperf/tcp highest 1-second throughput in its first ten seconds. For SNDa, the iperf results are 540 980 Mbps. For $S N D b$, the iperf results are 530 760 Mbps. For $S N D c$, the iperf results are 280 290 Mbps. The iperf results are consistent with our estimated capacity ratios. We guess that the $S N D a$ capacity is possibly $1 \mathrm{Gbps}$, and the $S N D b$ and $S N D c$ capacities are limited possibly by the virtual machine capability and by rate limiters (e.g., a token bucket shaper).

Note that PathComp sends out much less traffic than iperf. For example, PathComp sends less than 1 MBytes from SNDa, whereas iperf sends 65 117 MBytes just in the first second.

\section{CONCLUSIONS}

In this paper, we proposed a method called PathComp to relatively compare the capacities of two paths using the packet arrival sequence information instead of packet time information. In the future, we plan to extend PathComp to simultaneously compare more than two paths, and plan to relatively compare the available bandwidths of different paths.

\section{ACKNOWLEDGMENT}

The work reported in this paper is supported in part by NSF CNS-1017561.

\section{REFERENCES}

[1] Amazon Web Services, Inc. Amazon Elastic Compute Cloud. http: //aws.amazon.com/ec2/.

[2] R. Carter and M. Crovella. Measuring bottleneck link speed in packetswitched networks. Performance evaluation, 27:297-318, October 1996.

[3] L. Chen, T. Sun, B. Wang, M. Sanadidi, and M. Gerla. PBProbe: A capacity estimation tool for high speed networks. Computer Communications, 31(17):pp. 3883-3893, November 2008.

[4] L. Cheng and C. Wang. Defeating network jitter for virtual machines. In Proceedings of IEEE Conference on Utility and Cloud Computing (UCC), Melbourne, Australia, December 2011.

[5] D. Croce, E. Leonardi, and M. Mellia. Large scale available bandwidth measuremens: interference in current techniques. IEEE Transactions on Network and Service Management, 8(4):pp 361-374, December 2011.

[6] C. Dovrolis, P. Ramanathan, and D. Moore. Packet-dispersion techniques and a capacity-estimation methodology. IEEE/ACM Transactions on Networking, 12(6):963-977, December 2004.

[7] A. Downey. Using pathchar to estimate Internet link characteristics. In Proceedings of ACM SIGCOMM, pages 241-250, Cambridge, MA, August 1999.

[8] M. Jain and C. Dovrolis. End-to-end available bandwidth: measurement methodology, dynamics, and relation with TCP throughput. IEEE/ACM Transactions on Networking, 11(4):537-549, August 2003.

[9] G. Jin and B. Tierney. System capability effects on algorithms for network bandwidth measurement. In Proceedings of ACM IMC, Miami Beach, FL, October 2003.

[10] R. Kapoor, L. Chen, L. Lao, M. Gerla, and M. Y. Sanadidi. CapProbe: a simple and accurate capacity estimation technique. In Proceedings of ACM SIGCOMM, Portland, Oregon, August 2004.

[11] K. LaCurts, S. Deng, A. Goyal, and H. Balakrishnan. Choreo: Networkaware task placement for cloud applications. In Proceedings of ACM IMC, Barcelona, Spain, October 2013.

[12] K. Lai and M. Baker. Measuring link bandwidths using a deterministic model of packet delay. In Proceedings of ACM SIGCOMM, New York, NY, August 2000.

[13] Y. Liu, Y. Guo, and C. Liang. A survey on peer-to-peer video streaming systems. Journal of Peer-to-Peer Networking and Applications, 1(1):1828, March 2008.

[14] Microsoft. Introduction to receive side scaling. http://msdn.microsoft. com/en-us/library/windows/hardware/ff556942\%28v=vs.85\%29.aspx.

[15] Naval Research Laboratory. Multi-Generator (MGEN). http://www.nrl. navy.mil/itd/ncs/products/mgen.

[16] V. Paxson. End-to-end internet packet dynamics. In Proceedings of ACM SIGCOMM, France, September 1997.

[17] V. Paxson. On calibrating measurements of packet transit times. ACM SIGMETRICS Performance Evaluation Review, 26(1):pp 11-21, June 1998.

[18] PlanetLab. An open platform for developing, deploying, and accessing planetary-scale service, 2002. http://www.planet-lab.org/.

[19] R. Prasad, M. Jain, and C. Dovrolis. Effects of interrupt coalescence on network measurements. In Proceedings of PAM, France, April 2004.

[20] R. Prasad, M. Murray, C. Dovrolis, and K. Claffy. Bandwidth estimation: metrics, measurement techniques, and tools. IEEE Network, 17(6):2735, November-December 2003.

[21] J. Whiteaker, F. Schneider, and R. Teixeira. Explaining packet delays under virtualization. ACM SIGCOMM Computer Communication Review, 41(1):pp 39-44, January 2011. 